\title{
Feature Extraction Method for Wheat Diseases Based on Multi-fractal Spectrum
}

\author{
Feiyun Zhang \\ College of Electrical and Information Engineering \\ Xuchang University \\ Henan Xuchang, China 461000 \\ e-mail: ytyx_123@163.com
}

\begin{abstract}
Wheat diseases image noise was effectively removed using lifting scheme multi-wavelet transform and multi-fractal analysis, and then it used multi-fractal theory to segment diseases image and extract eight multi-fractal spectrum values as wheat shape feature of diseases. Experiments showed that the shape characteristic value of different wheat diseases had great difference, and the shape characteristic value of similar diseases had certain regularity. Therefore, it could extract shape characteristic value to recognize wheat diseases.
\end{abstract}

Keywords-Wheat diseases; Multi-wavelet transform; Multifractal spectrum; Feature extraction; shape feature

\section{INTRODUCTION}

In China, wheat is the second largest food crop after rice, which plays a significant role to meet people's needs and to ensure food security. While, every year, large area of wheat diseases seriously affect the yield and quality of the wheat. Traditional diagnostic methods of wheat disease mainly rely on the visual judgment of agricultural producers and researchers to identify the type of the disease, the severity and the affected regions.This identification method is subjective and prone to bias diagnostic results. Therefore, the research of wheat diseases intelligent identification has important social and economic significance.

Nowadays, many scholars have conducted the research on the image recognition method of crop diseases at home and abroad [1-7]. Most of the researches nowadays are based on the texture and color features to carry on the disease recognition in the visible area. These algorithms or methods are relatively complex, run longer and less than ideal identification. This paper introduced lifting scheme multi-wavelet transform and multi-fractal theory, extracted eight multi-fractal spectrum value as wheat shape feature of diseases. The experimental results showed that it could recognize wheat disease by extracting the shape feature values.

\section{ACQUISITION OF WHEAT DISEASE IMAGE}

The image of the article mainly comes from still pictures from a digital camera, primary color atlas of wheat diseases and insect pests [8] and network photo gallery. This study collects 200 pieces of images with different wheat disease parts and different growth stages. Besides, each disease includes 70 samples. The main wheat diseases Category are stripe rust, leaf rust and powdery mildew.
Wheat leaf disease pictures collected are color images, which has amounts of data affecting the speed of image processing. In order to improve the speed of image processing, it needs to convert a color image to gray scale image. According to the degree of sensitivity of the human eye on the three colors of R, G, B, we can use the weighted average to make the color image become gray, The conversion formula is:

$$
\text { Gray }=0.3 \times R+0.59 \times G+0.11 \times B
$$

Then, the gray-scale image is converted to binary image so that the computer can further process it. Wheat stripe rust disease artwork and conversion of gray scale images show in figure 1.

\section{MULTI-FRACTAL}

A. Image denoising based on lifting scheme multi-wavelet transform and multi-fractal analysis

As a branch of nonlinear science, the object of study of the fractal theory is generated by the non-linear system nondifferentiable and smooth complex geometry. Only a fractal dimension can not completely characterize complex geometric nature, so in order to characterize its comprehensive feature more than one dimension must be used at the same time. Multi-fractal is to use more than one dimension from global and local part to describe the overall characteristics of the complex geometry in nature. While, Fractal dimension not only can measure the degree of complexity of the image surface and irregular, but also it has multi-scale wavelet transform, multi-resolution transform invariance. Different angle of Multi-fractal theory and different describe when dealing with different problems leads to vague definition of multi-fractal theory in mathematics.

Multi-fractal analysis focuses on adjusting Multi-fractal parameters in accordance with the needs of the image features and image processing. It can be used $\alpha \sim f(\alpha)$ language to describe Multi-fractal theory, and $\alpha$ can be called hölder index, because it controls the probability density singularity and reflects sub-body small area local fractal dimension. Therefore, $\alpha$ is commonly known as the singularity exponent. $f(\alpha)$ Commonly refers to as multifractal spectrum is called multi-fractal singularity spectrum, which reflects the global information of complex fractal object. Different information has different singularity, so 
$(\alpha \sim f(\alpha)$ owns different distribution rule [9]. The distribution rule of $\alpha$ and $f(\alpha)$ are as followed:

a) In the not smooth regions of the image or the irregular regions of the signal, $f(\alpha<2)=2$ and $f(\alpha=2)<2$;

b) In the smooth regions of the image, $f(\alpha \approx 2) \approx 2$.

In image processing, the noise is seen as irregular nonsingular point. And the target image can be seen as a large number of singular points. At first, adjusting the value of $\alpha$ to make sure $f(\alpha)$ of all points as close as the maximum value of 2, Then transforming $f(\alpha)$, at the same time the relative intensity of multi-fractal spectrum does not change, that can achieve the image denoising. The disease image denoising algorithm combined multi-fractal analysis and lifting scheme of multi-wavelet transform are as followed:

a) Upgrade image using discrete Multi-wavelet decomposition algorithm, $W T_{x}(j, k)$ is its transform coefficient, $j$ denotes enhance format of Multi-wavelet transform, $k$ is the location of the wavelet coefficients.

b) Adjust the value of the singularity index $\alpha$ to make the value of $f(\alpha(q))$ close to $f_{\text {max }}(\alpha), \alpha$ denotes $\alpha$, Set $W T_{x}^{\prime}(j, k)=W T_{x}(j, k) \cdot 2^{-j\left(\alpha-\alpha^{\prime}\right)}$, that means lifting scheme wavelet transform coefficient varies with the changes of $\alpha$, thereby, $f(\alpha)$ is adjusted to the maximum.

c) According to $W T_{x}^{\prime}(j, k)=W T_{x}(j, k) \cdot 2^{-j\left(\alpha-\alpha^{\prime}\right)}$, we can calculate the adjusted lifting scheme Multi-wavelet transform coefficients. Then, the coefficient wavelet inverse transform algorithm is done to obtain denoising image, as showed on below Figure 3 (d).

From Figure 3, we can find that the denoising image using of multi-fractal analysis and lifting scheme wavelet transform, it not only be eliminated speckle noise completely, but also the change of image plane area be made smooth, and the clear image edge is visible. The experiments show that this kind of method is feasible.

\section{B. Local edge segmentation based on multifractal theory of image}

The purpose of image segmentation is to separate wheat leaf disease image and background so that it is convenient wheat leaf diseases contour features. Image segmentation based on multi-fractal theory implementation steps are as follows:

a) Calculate the image singularity exponent $\alpha$

Singularity exponent $\alpha$ represents the local information of the image. We definite $V(i)$ as $i \times i$ square area and $I(x, y)$ as the central point of $V(i)$, $\alpha(x, y)=\frac{\log \mu(V(i))}{\log (i)}(i=2 n+1, n=0,1, \cdots)$. The value of $i$ reflects the range of the image singularity. After experiments confirmed, the smaller the value $\boldsymbol{i}$, the more it can reflect the image of the local singularity and the greater the value $i$, the better reflect the wide range of image singularity.

b) Multi-fractal spectrum of computing image $f(\alpha)$

It can be used $f(\alpha)$ to describe the global information of the image. According to the singularity index calculated in the previous step, we can calculate the maximum value of $\alpha: \alpha_{\max }=\max (\alpha(i, j))$, and the minimum of $\alpha: \alpha_{\min }=\min (\alpha(i, j))$. Then we can divide $\left[\alpha_{\min }, \alpha_{\max }\right]$ into $\mathrm{N}$ parts and get singular values of each center of the area which will replace the value of the other points in the region. At last, Hausdorff spectrum can be used to calculate $f(\alpha)$.

c) According to the results obtained by the first two steps, we can select the appropriate maximum and minimum values with relative capacity and use multi-fractal theory to carry on local edge segmentation of wheat diseases image. Divided image is shown in Figure 2.

\section{Multi-fractal characteristic spectrum extraction of wheat disease}

According to the algorithm based on multi-fractal spectrum, we can complete multi-fractal spectrum calculation by Matlab programming, draw multi-fractal spectrum curve and calculate some important characteristic values on the multi-fractal spectrum curve, such as: the biggest value $\alpha_{\max }$ and the youngest value $\alpha_{\min }$ of Singularity index $\alpha$ and the relative $f\left(\alpha_{\max }\right)$ and $f\left(\alpha_{\text {min }}\right)$; Spectral width $\Delta \alpha=\alpha_{\text {max }}-\alpha_{\text {min }}$; Spectrum difference $\Delta f=f\left(\alpha_{\max }\right)-f\left(\alpha_{\min }\right) ; \alpha_{d}$ : the value of $\alpha$ enable $f(\alpha)$ achieve the biggest value; m: fitting curve of the first power coefficient, at the place of $\alpha_{d}$, using the method of least squares to carry on fitting curve, $f^{\prime}(\alpha)=l\left(\alpha-\alpha_{d}\right)^{2}+m\left(\alpha-\alpha_{d}\right)+n$ is used to describe the fitting quadratic function.

\section{EXPERIMENTS AND RESULTS}

Figure 4 shows corresponding multi-fractal spectrum curve of wheat stripe rust disease image, and Table 1 gives 8 multi-fractal spectrum characteristic values of wheat leaf disease image samples in the database.

Experiments showed that the shape characteristic value of different wheat diseases had great difference, and the shape characteristic value of similar diseases had certain regularity. Therefore, it could extract shape characteristic value to recognize wheat diseases.

\section{ACKNOWLEDGMENT}

This work was supported by the Science Research Project of Henan Education Department (Grant No. 12A510021), Science and technology R\&D program of 
Xuchang Science and technology bureau (Grant No.1101060).

\section{REFERENCES}

[1] R. Pydipati, T.F. Burks, W.S Lee. Identification of citrus disease using color texture features and discriminant analysis. Computers and electronics in agriculture, 2006, (52): 49-59.

[2] R. Pydipati, T.F. Burks, W.S Lee. Statistical and neural network classifiers for citrus disease detection using machine vision. Transactions of the ASAE, 2005, 48(5): 2007-2014.

[3] G.L. Xu, H.P. Mao, P.P. Li. Extracting color features of leaf color images. Transactions of CSAE, 2002, 18(4): 150-154. (in Chinese )

[4] Y. Lu , H.O. Guan, B.Zhao, et al. Sdudy on the method of image preprocessing and feature extraction for rice diseases. Journal of agricultural mechanization research, 2011(8): 27-30. ( in Chinese )

[5] Y.W.Tian, Y.Niu. Research on recognition of cucumber disease based on image processing in sunlight greenhouse. Journal of agricultural mchanization rsearch, 2006(2): 151-160. ( in Chinese )

[6] B.Q. Chen, X.M. Guo, X.H. Li. Image diagnosis algorithm of diseased wheat. Transactions of the chinese society for agricultural machinery, 2009, 40(12): 190-195. ( in Chinese )

[7] Y.W. Tian, C.S. Zhang, C.H. Li. Application of support vector machine to shape recognition of plant disease spot. Transactions of the chinese society for agricultural machinery, 2004, 20(3): 134-136. ( in Chinese )

[8] H.S. Shang, F.K. Wang, X.L. Li, et al. Primary color atlas of wheat diseases and insect pests. Beijing: Golden Shield Press, 2007. ( in Chinese )

[9] X.Q. Pu. Image recognition based on multi-fractal. Northwestern University master's thesis, 2009. ( in Chinese ) 


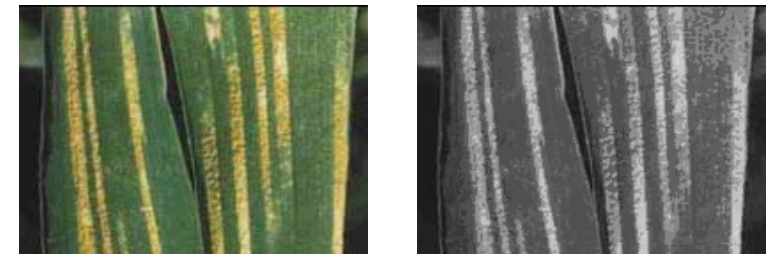

Figure 1. Wheat stripe rust disease artwork and conversion of gray scale images

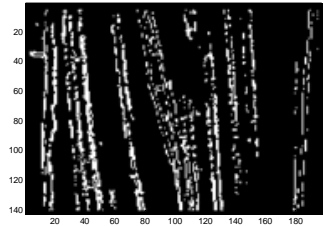

Figure 2. Image after Local segmentation

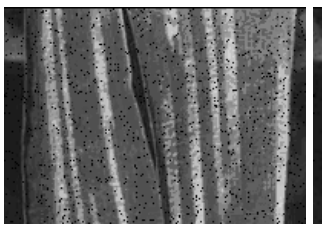

(a)

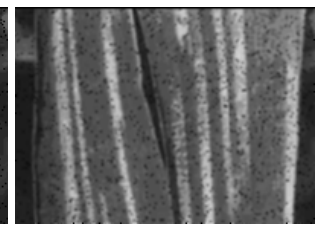

(b)

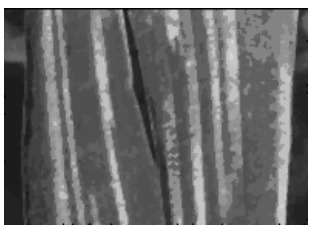

(c)

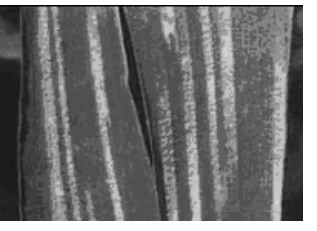

(d)

Figure 3. Experimental comparison figure of several denoising methods

(a) Disease image with noise (b) Median filtering denoising image (c) Field average filtering denoising image (d) Multiple fractal denoising image

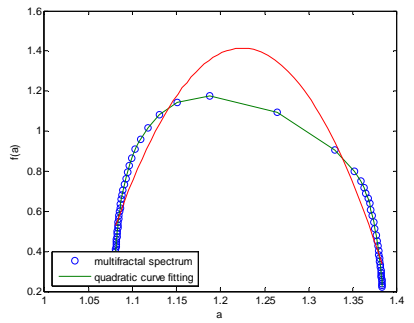

(a) Stripe rust blade

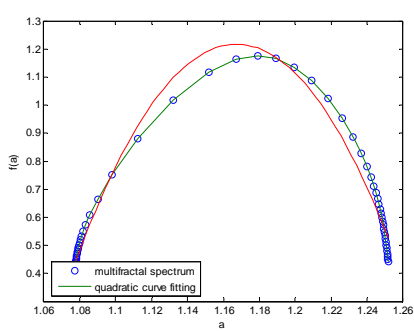

(b) Leaf rust leaves

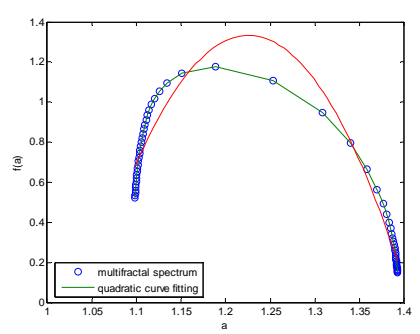

(c) Powdery mildew leaves

Figure 4. Corresponding multi-fractal spectrum curve of the wheat leaf disease image

Table I . 8 MULTI-FRACTAL SPECTRUM EIGENVALUE OF SOME WHEAT LEAF DISEASES IMAGE SAMPLES

\begin{tabular}{ccccccccc}
\hline \multirow{2}{*}{ Disease } & \multicolumn{7}{c}{ Multi-fractal spectrum eigenvalue } \\
\cline { 2 - 9 } & $\begin{array}{c}\text { Spectral } \\
\text { width }\end{array}$ & $\begin{array}{c}\text { Spectrum } \\
\text { difference }\end{array}$ & $\alpha_{\text {min }}$ & $\alpha_{\text {max }}$ & $f_{\text {min }}$ & $f_{\text {max }}$ & B & $\alpha_{d}$ \\
\hline $\begin{array}{c}\text { Stripe } \\
\text { rust 1 }\end{array}$ & 0.3025 & 0.17558 & 1.0807 & 1.3832 & 0.39975 & 0.22417 & 104.81 & 0.1897 \\
$\begin{array}{c}\text { Stripe } \\
\text { rust 2 }\end{array}$ & 0.3022 & 1.17553 & 1.0809 & 1.384 & 0.39971 & 0.2242 & 104.9 & 0.1891 \\
$\begin{array}{c}\text { Leaf rust1 } \\
\text { Leaf rust2 }\end{array}$ & 0.17486 & -0.04562 & 1.077 & 1.2518 & 0.3953 & 0.44092 & 225.14 & 1.179 \\
$\begin{array}{c}\text { Powdery } \\
\text { mildew 1 }\end{array}$ & 0.294 & 0.37242 & 1.0982 & 1.3922 & 0.52176 & 0.14933 & 99.284 & 1.1883 \\
$\begin{array}{c}\text { Powdery } \\
\text { mildew 2 }\end{array}$ & 0.296 & 0.3725 & 1.0986 & 1.3924 & 0.52184 & 0.14946 & 98.899 & 1.1881 \\
\hline
\end{tabular}

